\title{
Graphical Comparison of Image Analysis and Laser Diffraction Particle Size Analysis Data Obtained From the Measurements of Nonspherical Particle Systems
}

Submitted: May 18, 2005; Accepted: April 25, 2006; Published: August 18, 2006

Richard N. Kelly, ${ }^{1}$ Kimberly J. DiSante, ${ }^{2}$ Elliott Stranzl, ${ }^{1}$ Jacqueline A. Kazanjian, ${ }^{3}$ Paul Bowen, ${ }^{4}$ Tatsuyama Matsuyama, ${ }^{5}$ and Nadine Gabas ${ }^{6}$

${ }^{1}$ Johnson \& Johnson Pharmaceutical Research \& Development, LLC Spring House, PA

${ }^{2}$ University of Pittsburgh Department of Pharmacology Pittsburgh, PA

${ }^{3}$ Yves Rocher North America Inc, Exton, PA

${ }^{4}$ Powder Technology Laboratory, Materials Science and Engineering Department, Ecole Polytechnique Fédérale de Lausanne (EPFL), CH-1015 Lausanne, Switzerland

${ }^{5}$ Department of Environmental Engineering for Symbiosis, Soka University, 1-236 Tangi-cho, Hachioji, Tokyo, Japan

${ }^{6}$ Laboratoire de Génie Chimique, 5 Rue Paulin Talabot, 31106 Toulouse Cedex 01, France

\section{ABSTRACT}

The purpose of this paper is to describe results from the use of a set of Excel macros written to facilitate the comparison of image analysis (IA) and laser diffraction (LD) particle size analysis (psa) data. Measurements were made on particle systems of differing morphological characteristics including differing average aspect ratios, particle size distribution widths and modalities. The IA and LD psa data were plotted on the same graph treating both the weighting and the size unit of the LD psa data as unknowns. Congruency of the IA and LD plots was considered to indicate successful experimental determination of the weighting and size unit. The weighting of the resulting LD psa data (so-called volume-weighted) is shown to be better correlated with IA area-weighted data. The size unit of LD psa data is shown to be a function of particle shape. In the case of high aspect ratio particles characterized by approximately rectangular faces the LD psa data is shown to be a function of multiple particle dimensions being related to IA size descriptors through a simple variation of the law of mixtures. The results demonstrate that successful correlations between IA and LD psa data can be realized in the case of non-spherical particle systems even in the case of high aspect ratio particles; however, the inappropriateness of the application of the Equivalent Spherical Volume Diameter and the Random Particle Orientation assumptions to the interpretation of the LD psa results must first be acknowledged. Correlation permits cross validation of IA and LD psa results increasing confidence in the accuracy of the data from each orthogonal technique.

Corresponding Author: Richard N. Kelly, Johnson \& Johnson Pharmaceutical Research \& Development, LLC, Room P-1029 McKean \& Welsh Roads, Spring House, PA 19477-0776. Tel: (215) 628-5150; Fax: (215) 540-4684; E-mail: Rkelly@prdus.jnj.com
KEYWORDS: equivalent spherical volume diameter, Excel macros, image analysis, laser diffraction, nonspherical particles, particle size analysis, random particle orientation, results correlation.

\section{INTRODUCTION}

Image analysis (IA) and laser diffraction (LD) are among the most commonly employed particle size analysis (psa) techniques within the pharmaceutical industry.

Image analysis over the past decade has been transformed into a very affordable and powerful research tool as a result of advances in computer technology, digital imaging technology, and microscopy. The many modes of microscopy and photography available such as scanning electron microscopy (SEM), polarized light microscopy, fluorescence microscopy, stereomicroscopy, and stroboscopic photography allow the IA characterization of pharmaceutical particle systems of size ranging from hundreds of nanometers to several millimeters within simple and complex media such as creams and gels, often with little sample alteration, that may not be easily characterized by any other method. ${ }^{1}$

However, IA results can be particularly vulnerable to sampling errors, as only a few thousand particles are typically included in an analysis. Also IA particle size analysis can be characterized by relatively long analysis times, with analysis times exceeding 15 minutes not being uncommon. Another limitation with IA is that of working with a 2-D projected image of a 3-D particle, which is not always simple to interpret.

Laser diffraction particle size analysis (LD psa), in contrast, is characterized by highly reproducible results and relatively short analysis times, with the typical analysis time being less than 1 minute. $^{2}$ For these reasons, LD particle size analysis is arguably the most popular particle size analysis technique within the pharmaceutical industry, especially within quality control laboratories. 
LD particle size analysis is an ensemble technique with a recognized dependence upon particle shape as well as particle size. Especially in the case of nonspherical particle systems, it is dangerous to attempt the interpretation of the results from an LD particle size analysis experiment without morphological data. The success of LD-based quality control (QC) particle size analysis (psa) test methods then relies on the meaningful correlation of results obtained from other psa techniques. Guidance documents ${ }^{3-5}$ often suggest the use of IA, which for the purpose of this article will be considered a computer-aided form of microscopy. Microscopy is generally considered the most reliable referee psa technique as it allows direct visualization of the sample under investigation. Unfortunately, the guidance documents do not include specific instructions on how to meaningfully conduct the quantitative data comparison.

As revealed from work conducted using the AEA shape standards available from LGC Promochem (Teddington, UK; www.lgcpromochem.com $)^{6}$ and a review of the literature, 2 primary reasons appear to exist for the failure to correlate IA and LD psa results from the measurements of nonspherical particles.

The first reason appears to be that previous investigators ${ }^{7-13}$ chose only a single size descriptor from the many available from IA. The most common size descriptors chosen were based on the equivalent circular area diameter (ECAD) or the equivalent spherical volume diameter (ESVD). It has been known since the work of Gabas et $\mathrm{al}^{7}$ that for nonspherical particles, the interpretation of the diffraction events occurring from each of the chords that can be drawn across the cross-sectional area projection of a particle (Feret diameters) does not lead to a single description of ESVD or ECAD. In reality, the LD psa data, even for mono-sized nonspherical test samples, are a combination of particle breadth, longest dimension, and eventually other particle dimensional measurements for more complex shapes. Therefore, attempts to correlate IA and LD psa data using a single IA size descriptor did not meet with general success.

The second reason for the failure appears to be that graphical analysis routines that would have permitted the facile comparison of IA psa data with the corresponding LD data have been, and still are generally, absent from IA software packages. The expectation of IA software and system providers seems to be that analysts would either export IA data to other software or use the tools included with the IA software to create the graphical analysis routines needed to compensate for the deficiency within the manufacturer's software. Yet it appears that the needed graphical analysis tools have either not been created or have not been made available to the community of particle size analysts.

These observations prompted the creation of a set of Excel macros that allow the reintegration of LD psa data, the se- lection of the appropriate IA probability basis (number or area), the selection of the IA size descriptors (eg, breadth, equivalent circular area, longest dimension), and the plotting of subsets of the IA data chosen on the basis of user-selected criteria. The observations further prompted the application of the Excel macro set to the correlation of the LD and IA psa data from particle systems containing approximately rectangular/acicular, plate-like/disc and other shaped particles.

\section{EXPERIMENTAL}

\section{Samples}

The following samples and others were analyzed by both IA and static LD:

1. Johnson \& Johnson Pharmaceutical Research \& Development (JJPRD) test samples of various morphologies,

2. nitrofurantoin purchased from Aldrich-Sigma (N7878100G, batch no. 042K1272, St Louis, MO), and

3. mica samples generously donated by Ogelbay Norton Specialty Minerals (C-3000, lot 10823, Kings Mountain, NC).

Images of these samples are presented in Figure 1.

\section{Instruments}

Image Analysis

Princeton Gamma-Tech Imix-PC

- Nikon Microphot FXA compound microscope interfaced with a Hamamatsu C2400 CCCD camera (Hamamatsu City, Japan).

- Leica MZ APO (Bensheim, Germany) stereomicroscope interfaced with an Optronics Engineering DEI750 CCD camera (Optronics, Goleta, CA).

\section{Laser Diffraction}

Laser diffraction was performed using a Beckman-Coulter LS 13320 (Fullerton, CA) configured with either the universal liquid module or the micro liquid module. Run conditions were as follows: sample refractive index, 1.50 to 1.60; circulation speed, $50 \%$; measurement time, 1 minute; and carrier fluid, either water or silicone oil (refractive index, 1.33 or 1.44 , respectively).

\section{Procedure}

IA psa and LD psa measurements were made on each of the samples, and the resultant data were plotted and relationships sought via application of the set of Excel macros (Macro set will be made available upon request). 
AAPS PharmSciTech 2006; 7 (3) Article 69 (http://www.aapspharmscitech.org).

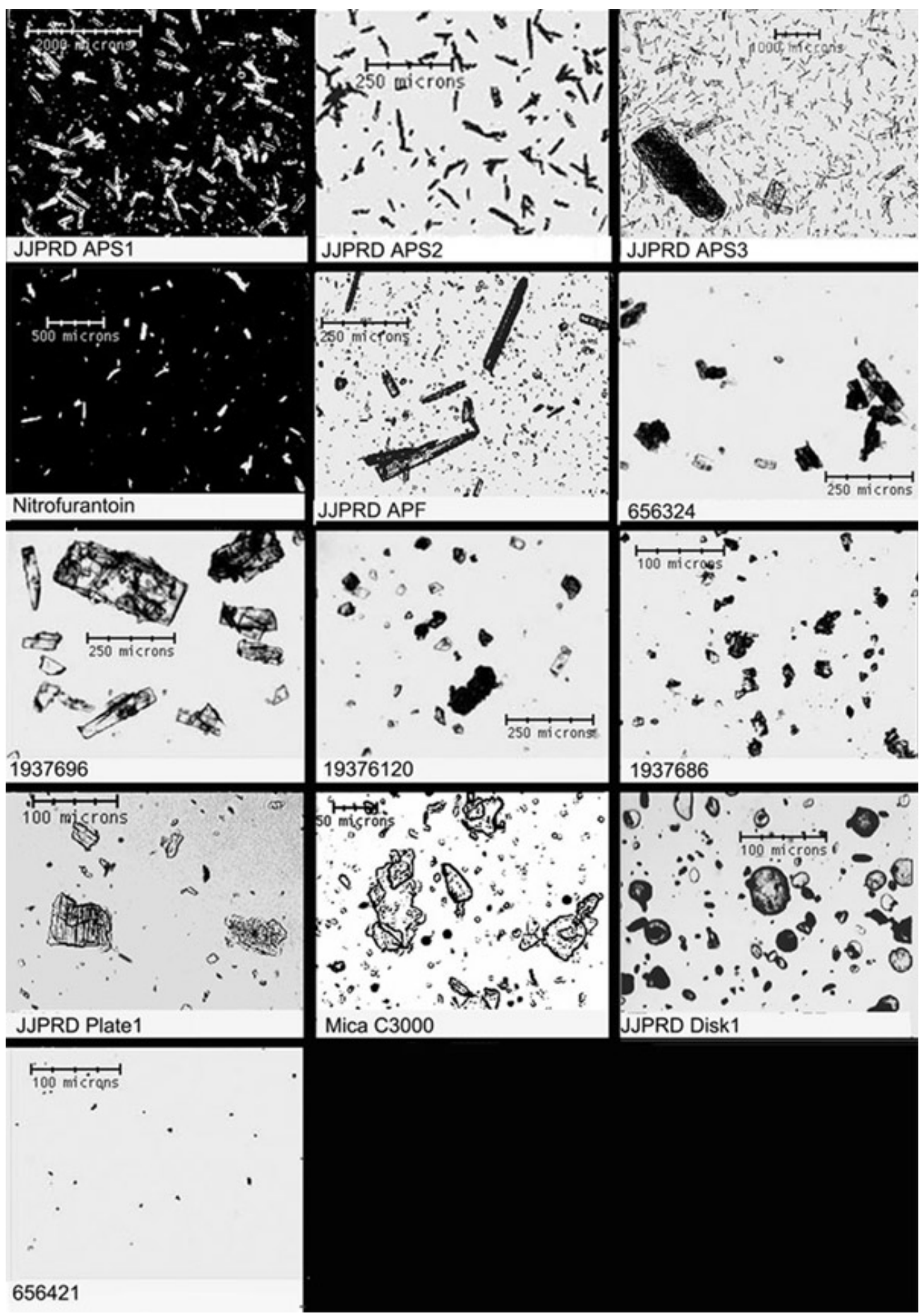

Figure 1. Photomicrographs of study samples.

\section{RESULTS AND DISCUSSION}

The Excel macro set consists of 2 functional units. The first unit plots directly measured and calculated IA measurements such as breadth (B), longest dimension (LDim), ECAD, and AR on both a cumulative number and cumulative area percentage (CAP) basis. The $\mathrm{x}$-axis is simply size in micrometers (ie, no attempt is made to interpret the abscissa in terms of a specific size descriptor). This unit includes a filter dialog box to permit plotting of the IA data according to user- selected feature criteria. The second functional unit is used to plot the LD number percentage and cumulative volume percentage $(\% \mathrm{~V})$ measurement data on the same graph as the previously plotted IA data. A dialog box is opened upon activation of this unit that allows the LD data to be reintegrated and plotted.

Table 1 presents particle size and morphological data describing the powder samples included in this study. The AR of the test samples ranged from 1.42 to 2.42 . The size (longest dimension) range of the samples spanned from $20 \mu \mathrm{m}$ (656421b) to almost $2 \mathrm{~mm}$ in the cases of approximately 
AAPS PharmSciTech 2006; 7 (3) Article 69 (http://www.aapspharmscitech.org).

Table 1. Particle Size and Morphological Characterization Data*

\begin{tabular}{|c|c|c|c|c|c|c|c|}
\hline SAMPLE & CAP@AR = 2 & Break Point & $\begin{array}{l}\text { Trend Line } \\
\text { LDim vs AR }\end{array}$ & Mean AR & Range LDim & Range B & $\begin{array}{l}\text { IA No. } \\
\text { Particles }\end{array}$ \\
\hline APS2 & 21.29 & 20.99 & $0.0170 X+1.87$ & $2.42 \pm 1.01$ & 262 & 60 & 1239 \\
\hline APS1 & 34.18 & 38.40 & $0.0018 X+2.06$ & $2.03 \pm 0.77$ & 1110 & 577 & 10440 \\
\hline APS3 & 35.16 & 31.30 & $0.0010 X+2.49$ & $2.24 \pm 0.85$ & 1851 & 832 & 12738 \\
\hline Nitrofurantoin & 38.09 & 40.49 & $0.0080 X+1.85$ & $2.06 \pm 0.87$ & 344 & 123 & 1897 \\
\hline \multirow[t]{2}{*}{ APF $A R>2$} & 56.61 & 60.13 & $0.0102 X+1.62$ & $1.54 \pm 0.47$ & 434 & 94 & 7265 \\
\hline & & & & $2.60 \pm 0.63$ & 434 & 94 & 702 \\
\hline SCIO656324 & 56.89 & 66.90 & $0.0070 X+1.51$ & $1.69 \pm 0.57$ & 227 & 93 & 1208 \\
\hline SCIO1937696a & 61.50 & 57.21 & $0.0004 X+1.96$ & $1.94 \pm 0.87$ & 426 & 198 & 1337 \\
\hline Plate1 & 85.10 & Not Detected & $0.0008 X+1.92$ & $1.75 \pm 0.63$ & 108 & 75 & 1406 \\
\hline SCIO19376120a & 86.37 & Not Detected & $0.0020 X+1.54$ & $1.57 \pm 0.44$ & 199 & 129 & 1119 \\
\hline Mica C3000 & 88.55 & Not Detected & $0.0012 X+1.65$ & $1.42 \pm 0.33$ & 195 & 126 & 10429 \\
\hline Disk 1 & 96.20 & Not Detected & $-0.0036 X+1.58$ & $1.50 \pm 0.45$ & 82 & 59 & 1967 \\
\hline SCIO656421b & 96.70 & Not Detected & $0.0159 X+1.41$ & $1.48 \pm 0.27$ & 21 & 16 & 968 \\
\hline SCIO1937686b & 97.00 & Not Detected & $0.0009 X+1.46$ & $1.47 \pm 0.34$ & 71 & 44 & 1333 \\
\hline
\end{tabular}

*CAP indicates cumulative area percentage; AR, aspect ratio; LDim, longest dimension; B, breadth; IA, image analysis; APS, approximately rectangular particle sample; and APF, approximately rectangular sample with fines.

rectangular particle samples 1 and 3 (APS1 and APS3). Application of the macro set to the LD and IA data collected from the measurement of these samples revealed the relationship between IA and LD data to be primarily a function of AR, followed by modality. The results will therefore be discussed in 2 sections: First the results of the high AR $(>2)$ materials, followed by the results of the low AR $(<2)$ materials.

\section{AR Greater Than 2: Rectangular Particles}

Figure 2 presents examples of plots resulting from the application of the Excel macro set to IA and LD measurement data collected from measurements performed on particle systems characterized by average AR greater than 2 .

JJPRD ASP1 (Figure 2A) is monomodal, and JJPRD approximately rectangular sample with fines (APF) (Figure $2 \mathrm{~B}$ ) is bimodal. The data content of the plots has been reduced to facilitate visualization of relationships between the LD and IA data sets. The general points to be noted from these figures are the following:

1. LD \%V and IA CAP data can be successfully correlated for high $\mathrm{AR}(>2)$ materials.

2. Multiple image analysis size descriptors are needed for the correlation.

3. The so-called LD \%V data must be plotted on the same graph as the CAP IA data.

4. The abscissa cannot be interpreted in terms of ESVD.

5. Multimodal samples need to be deconvoluted into their respective modes for successful correlation.

6. The known limitations of each technique must be accounted for (eg, the insensitivity of LD to fines in particle systems characterized by broad size distributions).

\section{Multiple Size Descriptors}

In addition to the complete $\mathrm{LD} \% \mathrm{~V}$ data set, the data resulting from the reintegration of this data between the limits of ca. $38 \%$ to $100 \%$ is also plotted in Figure 2A. From this graph it is seen that the plot of the complete LD data set is first coincident to ca $38 \%$ CAP with the plot of the IA breadth plot, and then the IA longest dimension plot at $\sim 90 \%$ CAP. The reintegrated LD $\% \mathrm{~V}$ data are seen to be coincident with the IA longest dimension plot over the range of ca $50 \%$ to $100 \%$ CAP.

The relationship between IA and LD data appears to be discontinuous in the case of measurements made on materials characterized by ARs significantly greater than 1 . Initially, one graphical relationship between IA and LD data appears to exist in the "CAP versus Size" plot, and a second relationship appears to exist after a certain point. A comparison of the data contained in Figures 3, 4 and 5 and summarized in Table 1 indicates that the divergence point is size independent and is related to that point in the "CAP versus AR" plot, where the AR value exceeds 2 .

The simplest continuous function roughly describing this relationship is given by Equation 1:

$\% V(i)=\left(\left(A_{\text {minor chord }}(i)+A_{\text {major chord }}(i)\right) /\left(2 * A_{\text {total }}\right)\right) * 100+\mathrm{C}$,

where $\% \mathrm{~V}(\mathrm{i})$ is the $\mathrm{LD}$ cumulative volume percentage to size (i); A minor chord (i) and $\mathrm{A}$ major chord are the IA cumulative areas to size (i) corresponding to the respective CAP versus minor chord and CAP versus major chord plots; $\mathrm{A}_{\text {total }}$ is the total projected surface area of the particles included in the IA test; and $\mathrm{C}$ is a correction term needed to compensate for incomplete data collection during IA, use of an incorrect optical model during LD, the possible use by 
AAPS PharmSciTech 2006; 7 (3) Article 69 (http://www.aapspharmscitech.org).
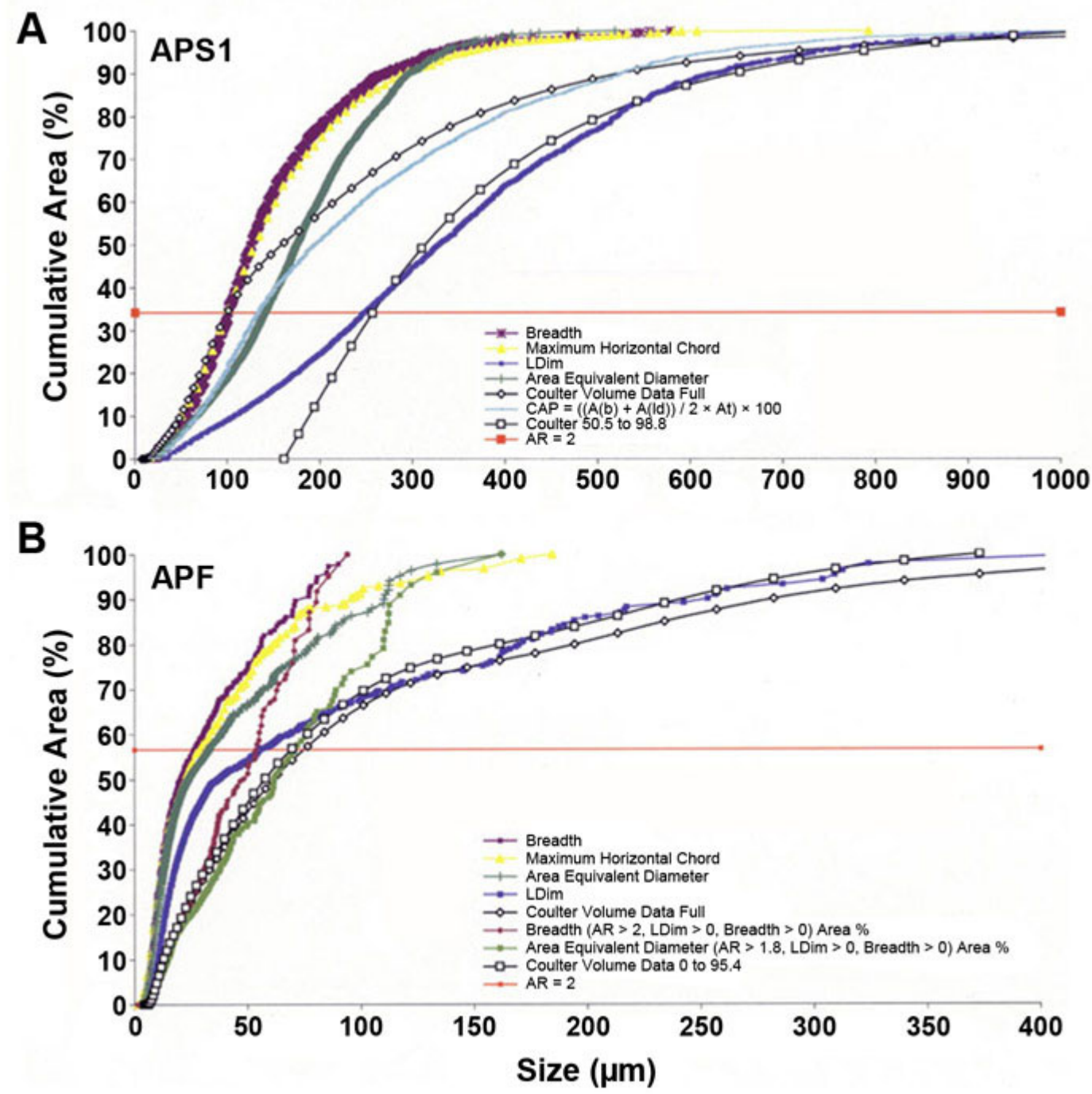

Figure 2. For an approximately rectangular monomodal, polydisperse, high-AR (>2) sample: a typical LD \%V and IA plot for APS1 and $\mathrm{a} \% \mathrm{~V}$ and IA plot showing influence of bimodality for APF.

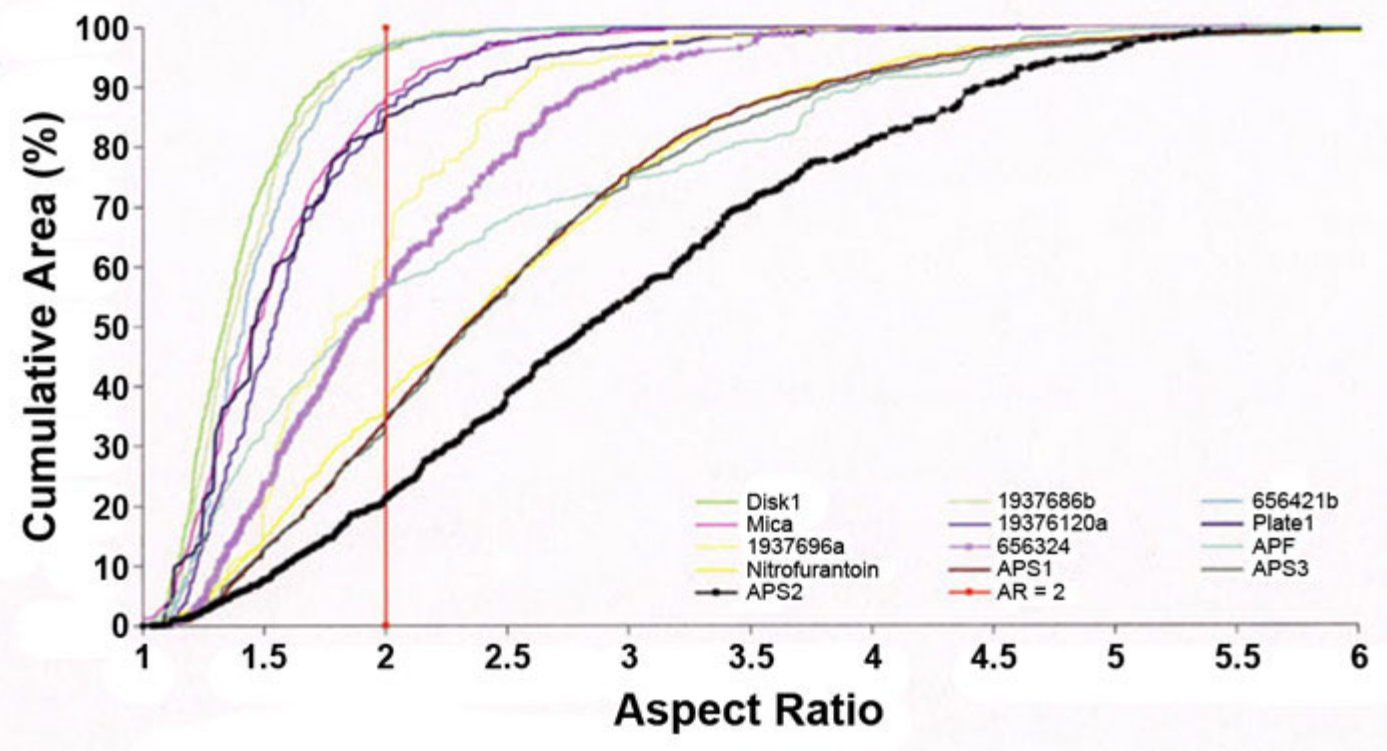

Figure 3. CAP versus AR plot showing study sample AR distributions. 
AAPS PharmSciTech 2006; 7 (3) Article 69 (http://www.aapspharmscitech.org).

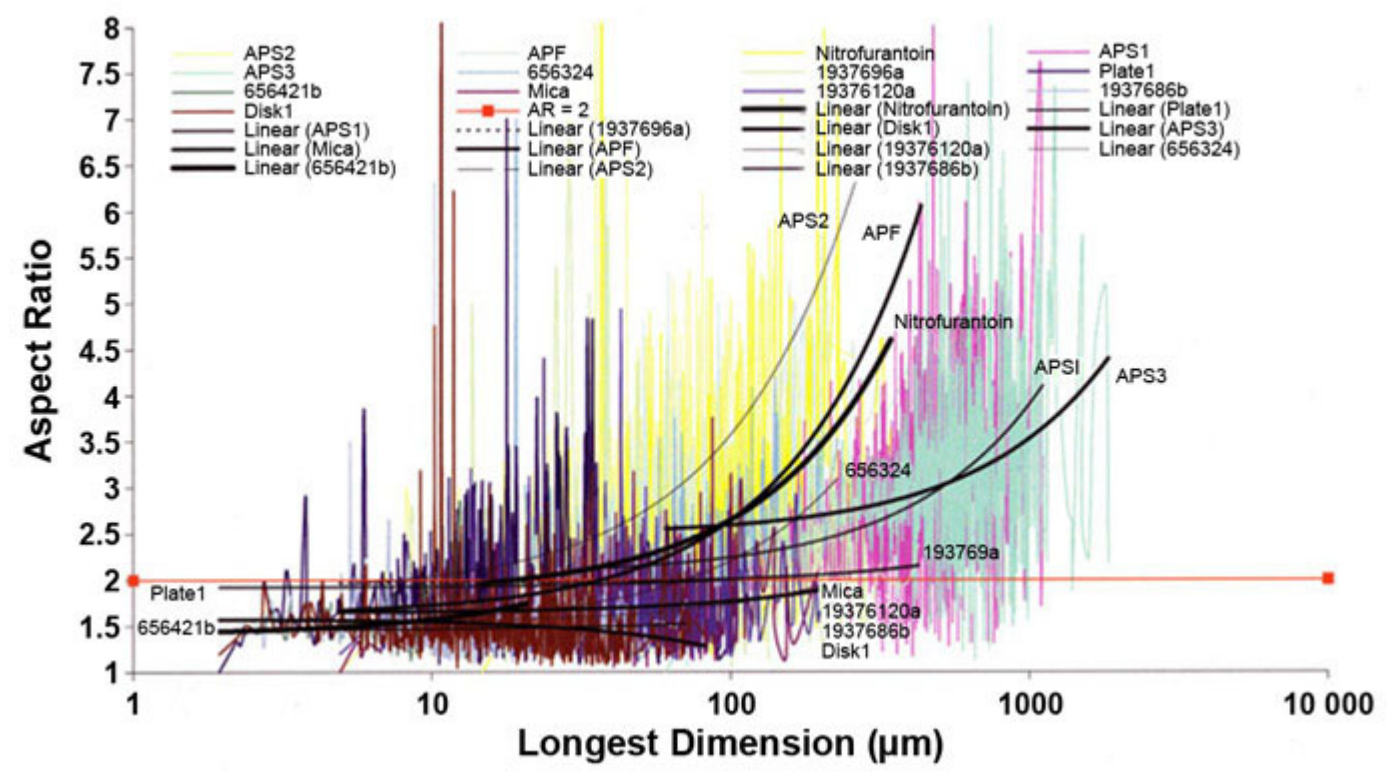

Figure 4. AR versus LDim plot showing study sample AR size dependence and distribution.

some manufacturers of arbitrary estimates of particle thickness, and other possible sources of systematic errors. This equation implies that each particle can be conceptually divided into 2 diffracting particles in which one is assigned the size of the minor chord and the second is assigned the size of the major chord, and the same projected surface area characterizes each. Equation 1 rewritten in terms of fractions takes the form:

$f_{L D, V}(\mathrm{i})=f_{\text {IA_Area, minor chord }}(\mathrm{i})+f_{\mathrm{IA} \_ \text {Area, major chord }}(\mathrm{i})+C$

In the case of approximately rectangular, polydisperse, and monomodal high AR materials such as APS1, the minor and major chords are, respectively, best described by image analysis breadth and longest dimension measurements (see Figure 2). In the case of monomodal powders characterized by average ARs greater than 2, and/or when the CAP value at which $\mathrm{AR}=2$ is less than $60 \%$, plots characterized by apparent bimodality as seen in the case of APS1 are generated. As the average AR value approaches 1, this function tends toward ECAD.

The applicability of Equation 1 cannot be extrapolated from the behavior of monodisperse materials of equivalent $\mathrm{AR}$ value as that of any given polydisperse sample. Figure $6 \mathrm{~A}$ is a photo of LGC Promochem AEA1003 shape standards having dimensions of $1 \times 1.7 \times 12 \mu \mathrm{m}$. Figure $6 \mathrm{~B}$ is the volume probability graph resulting from the measurement

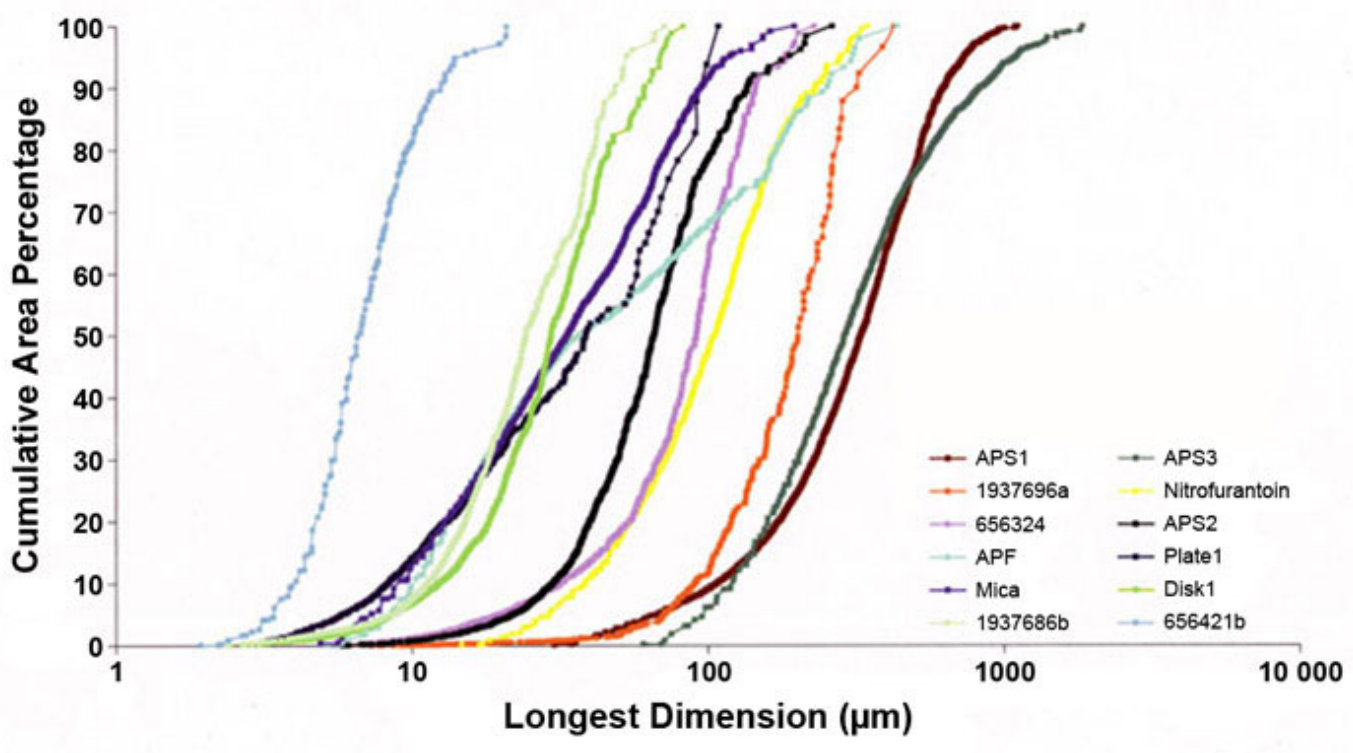

Figure 5. CAP versus LDim plot showing study sample size distributions. 

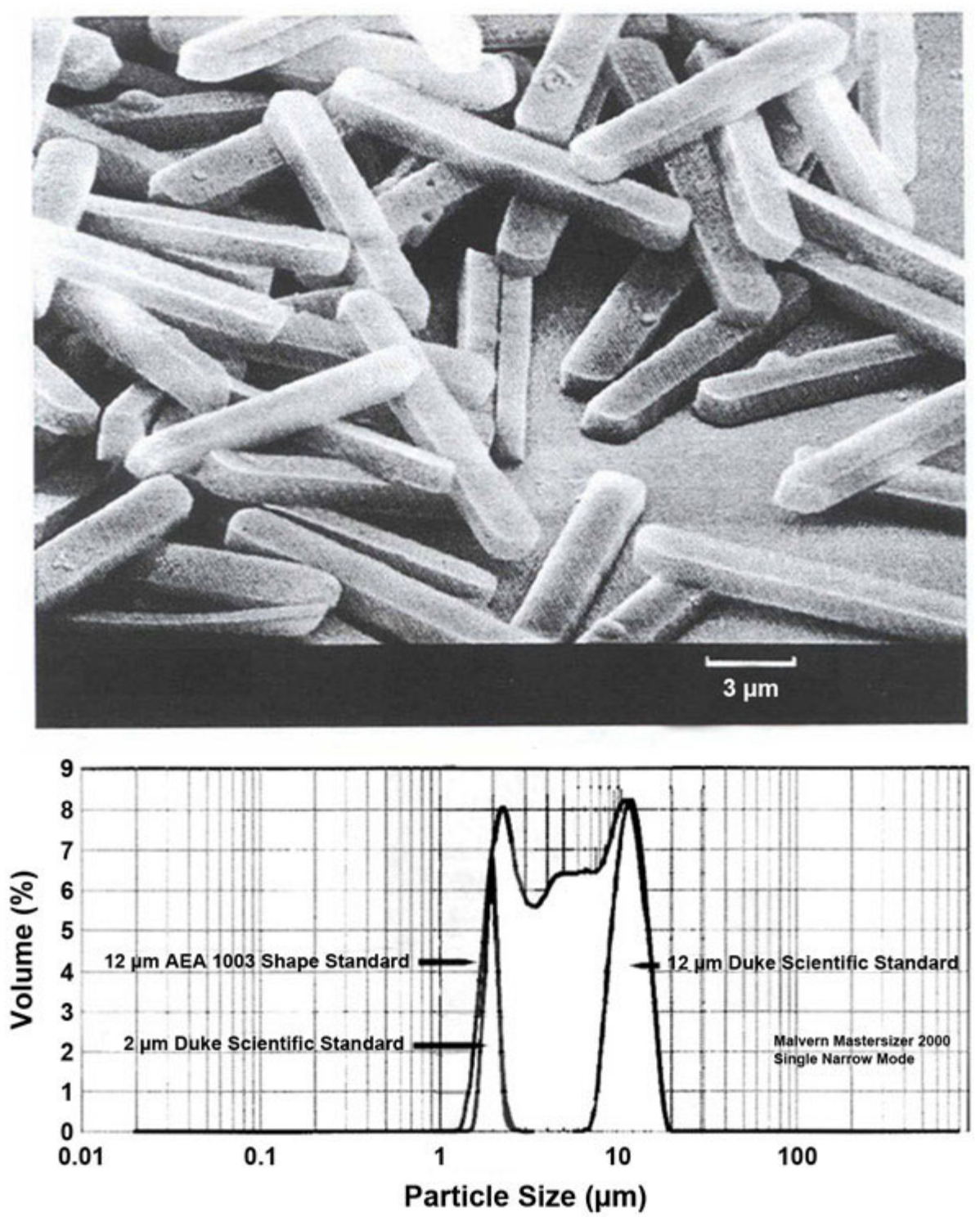

Figure 6. Photomicrograph of AEA 1003 shape standard particles and a typical LD \%V plot from their measurement.

of this standard. Whereas Equation 1 would call for 2 points located at $1.7 \mu \mathrm{m}$ (breadth) and $\sim 12 \mu \mathrm{m}$ (longest dimension) in the description of the LD behavior in terms of IA size descriptors, the actual plot is that of an apparent bimodal distribution as predicted by Matsuyama et al. ${ }^{14}$ The apparent bimodality is a result of the convolution of the multiple contributions to the LD data that are dominated by the breadth and length contributions in the case of approximately rectangular particles (as indicated for monodispersed rods by Gabas et $\mathrm{al}^{7}$ ). This apparent bimodal distribution is not easily described in terms of a small number of image analysis size descriptors.

The relationship between $\% \mathrm{~V}(\mathrm{i})$ and $\mathrm{A}(\mathrm{i})$ would require many factors indicated in Equation 3:

$$
\% V(i)=\left(\sum_{s=1}^{m} \sum_{d=1}^{n} A_{s d}(i) / \sum_{s=1}^{m} n_{s} * A_{s} \text { total }\right) * 100
$$

In this equation, $\mathrm{s}$ represents the number of contributing units into which a particle characterized by a complicated shape can be separated to provide the least number of contributing basic geometric forms from which the particle shape can be reconstructed, and $\mathrm{n}$ represents the least number of dimensional vectors, $\mathrm{d}$, required to describe the projected surface area of the component nonspherical basic geometric form(s). A(i) is the area over which diffraction occurs for a set of adjacent points having a similarly valued maximum chord length. For monodisperse samples, n appears to correspond to each unique chord that can be drawn across the projected particle surface.

Therefore, success would not be predicted in the attempt to use the results from LD measurements of monodisperse shape standards as a basis to project the required number of image analysis components needed to describe the LD data obtained from the measurement of a polydisperse particle 
system also characterized by shape inhomogeneity. The extent to which Equation 1 successfully describes LD measurement response for such systems is speculated to result from LD system noise filters within the analysis algorithms. The noise filters appear to filter all but the maxima from the apparent bimodal distributions. In Figure 6B, this would essentially correspond to elimination of all data below $8 \%$ differential volume frequency.

\section{Cumulative Area Percentage Versus Cumulative Volume Percentage}

Coincidence of the LD \%V data with the CAP IA data indicates that the best assignment of the LD plot ordinate in the case of approximately rectangular particles within the Fraunhofer domain would be "area" percent. This conclusion is in agreement with those of various studies contained in the literature. ${ }^{8,11,12,15-18}$ For example, Brewer and Ramsland $^{8}$ compared IA and LD psa results from particle systems characterized by spherical, plate-like, and needlelike morphologies. In their study, the IA CAP data, not to be confused with its ECAD transformation, were numerically compared with the LD cumulative $\% \mathrm{~V}$ data. In the cases of the spherical and plate-like particles, strong positive correlations between the IA psa area distribution $\mathrm{d}_{\mathrm{a}} 10, \mathrm{~d}_{\mathrm{a}} 50$, and $d_{\mathrm{a}} 90$ values and the LD psa volume distribution $\mathrm{d}_{\mathrm{v}} 10, \mathrm{~d}_{\mathrm{v}} 50$, and $d_{v} 90$ values were established. For the needle-like particles, the LD volume distribution values were smaller than the IA area distribution values. These conclusions with the results from Figure 2A can only be true if the approximately rectangular particles are aligned perpendicular to the laser beam. ${ }^{10}$

Furthermore, evidence exists to support the conclusion that flow orientation of particles within the measurement cells of LD psa systems is the rule rather than the exception. Berthold et $\mathrm{al}^{19}$ demonstrated in 2000 that the flow within LD psa systems in general has to be laminar to prevent the formation of bubbles. They also demonstrated that this laminar flow condition has the effect of orienting fiber-like particles in the direction of the flow. Recently conducted experimental and theoretical studies ${ }^{20,21}$ on the orientation of solids in laminar flow pipes have shown that laminar flow conditions characterized by even modest Reynold's numbers $(>100)$ result in the flow orientation of particles with little dependence on particle AR or density. Therefore, as particles are flow oriented, only the cross-sectional areas of primarily single faces of the particles are measured during the particles' transit through the flow cell and the resulting ensemble data, as shown by Gabas et al, ${ }^{7}$ cannot be successfully translated into an ESVD distribution measurement when the criteria for the valid application of the Fraunhofer theory are met.
The IA CAP/LD \%V correlation can also be understood from a consideration of the following facts: It is stated in International Organization for Standardization ISO $13320^{3}$ that "for medium sized particles (1-50 $\mu \mathrm{m})$ with $\mathrm{n}_{\mathrm{p}} / \mathrm{n}_{\mathrm{m}}>1,1$ and/or $\mathrm{k}_{\mathrm{p}}>0,05$ the Fraunhofer approximation usually also gives good results"; where $n_{p}$ stands for the real part of the refractive index of the particle, $n_{m}$ stands for the refractive index of the medium (ie, the carrier fluid), and $\mathrm{k}_{\mathrm{p}}$ stands for the imaginary part of the refractive index of the particle. It is known, as presented by Rawle, ${ }^{22}$ that "the Fraunhofer approximation... is a projected area prediction." Finally, as indicated by Kippax, ${ }^{23}$ "the refractive index of pharmaceuticals is generally in the range of 1.38-1.65." Therefore, for most pharmaceuticals, as they meet the criteria specified in ISO 13320 for the generally valid application of the Fraunhofer theory, a projected area prediction of the particle size distribution of the typical pharmaceutical is obtained from the application of LD psa systems whether the Mie theory or the Fraunhofer theory is applied.

\section{Bimodality and Measurement System Corrections}

APF is an example of a particle system that is bimodal and in which the larger particle size mode has a breadth-length overlap (Figure 2B). An initial inspection of the data shows less correspondence between the $\mathrm{LD} \% \mathrm{~V}$ than in the case of APS1 even for the area percentage IA longest dimension plot. However, user selection of only those data corresponding to particles characterized by ARs greater than 2 gives similar results to APS1. For this reduced data $(A R>2)$ the initial segment of the plot of the $\mathrm{LD} \% \mathrm{~V}$ data is seen to be coincident with the IA area percentage breadth (and area equivalent diameter data) and then the IA area percentage longest dimension as for APS1.

The need to reject the data from the IA measurement of the fines to achieve coincidence with the LD data indicates that the LD measurement was relatively insensitive to the presence of the fines. This finding is not unexpected as LD intensity is a function of the square of particle cross-sectional area. ${ }^{3}$ This may be accentuated for approximately rectangular particles when the breadth distribution of the larger mode overlaps the particle size distribution (PSD) of the fines rendering the intensity contribution from the fines indistinguishable from total intensity fluctuation. This would also provide an explanation for the observation made by Prasanna et $\mathrm{al}^{24}$ in a comparative study in which the performance of ensemble psa techniques (eg, LD) and particle counting techniques such as IA were reported. For sieve fractions of needle-like nitrofurantoin particles, they observed, "the large population of fines was only detected by the particle counting techniques and not by the ensemble technique." 
AAPS PharmSciTech 2006; 7 (3) Article 69 (http://www.aapspharmscitech.org).

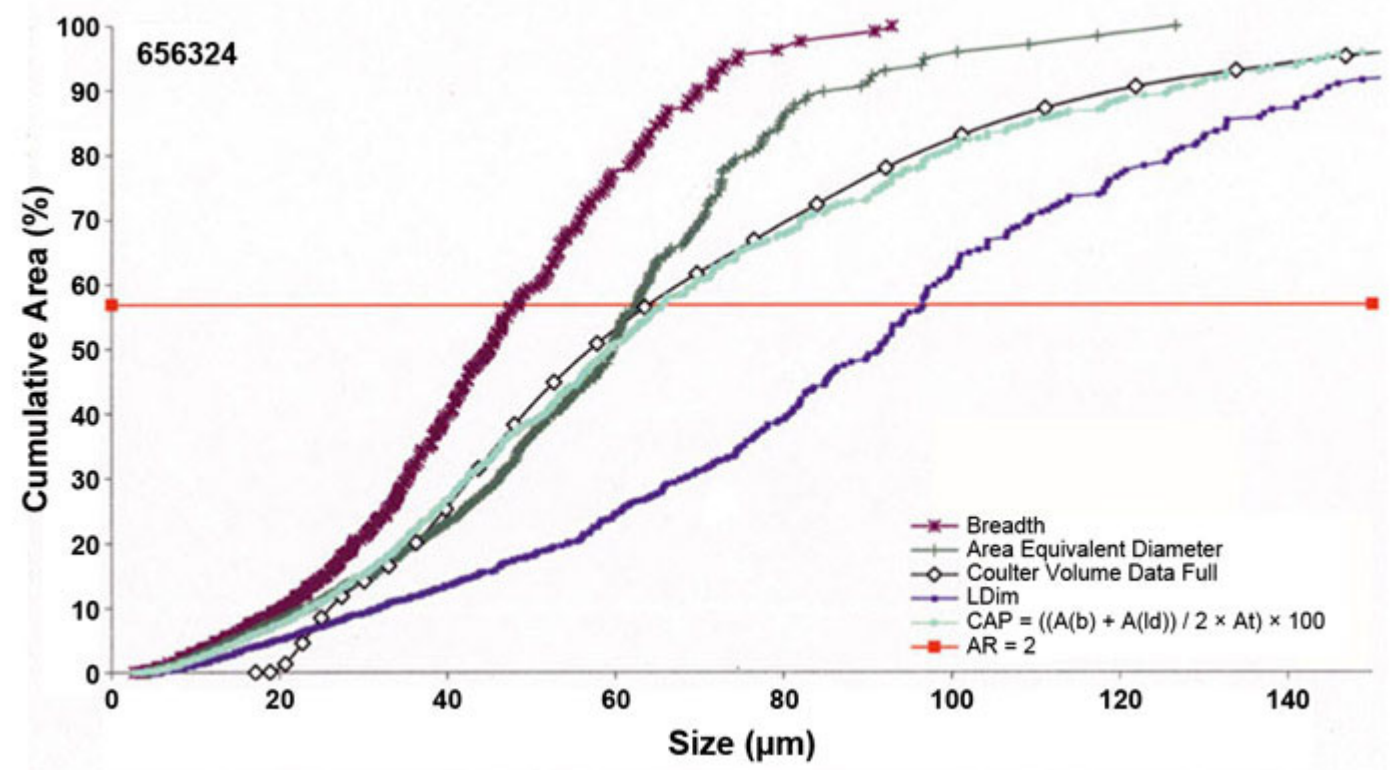

Figure 7. Example 1 of LD $\% \mathrm{~V}$ and IA plot for an approximately rectangular monomodal, polydisperse, medium-AR $(1.5<\mathrm{AR}<2)$ sample (656324).

\section{AR Less Than 2: Rectangular Particles}

Figures 7, 8, 9 and 10 show that for rectangular particles of average AR less than 2, Equation 1 more closely describes the IA/LD relationship than for particles with AR greater than 2. This is seen to be independent of particle size distribution width. As in the case of samples 1937696 and 656421 , it may be necessary to correct the data for data acquisition errors, sample preparation errors, etc. During the IA data collection of 656421 , for example, a magnification was employed that did not permit particles less than $2 \mu \mathrm{m}$ in length to be included in the data analysis. As the sample's particle size distribution, as correctly indicated by the results of the LD measurement data, extended below $1 \mu \mathrm{m}$, a correction factor of ca 2 (addition) had to be applied to the data to allow comparison. Confidence in the appropriateness of the correction step is obtained from the observation that the corrected data allow the LD/IA relationship to be described by Equation 1. Again it should be noted that Equation 1 results in curves that are initially coincident with the ECAD data plots but diverge after ca $50 \%$ to $60 \%$ CAP.

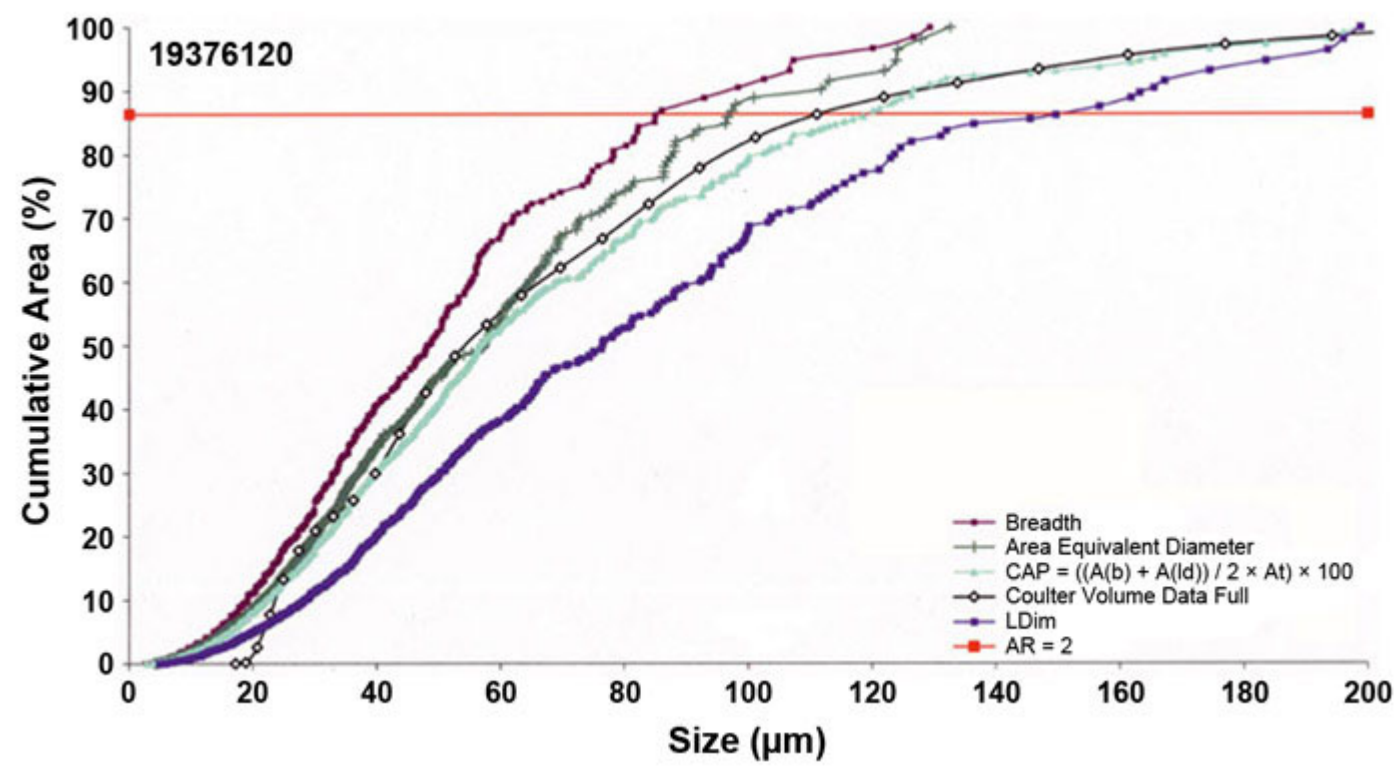

Figure 8. Example 2 of $\mathrm{LD} \% \mathrm{~V}$ and IA plot for an approximately rectangular monomodal, polydisperse, medium-AR $(1.5<\mathrm{AR}<2)$ sample (19376120a). 
AAPS PharmSciTech 2006; 7 (3) Article 69 (http://www.aapspharmscitech.org).

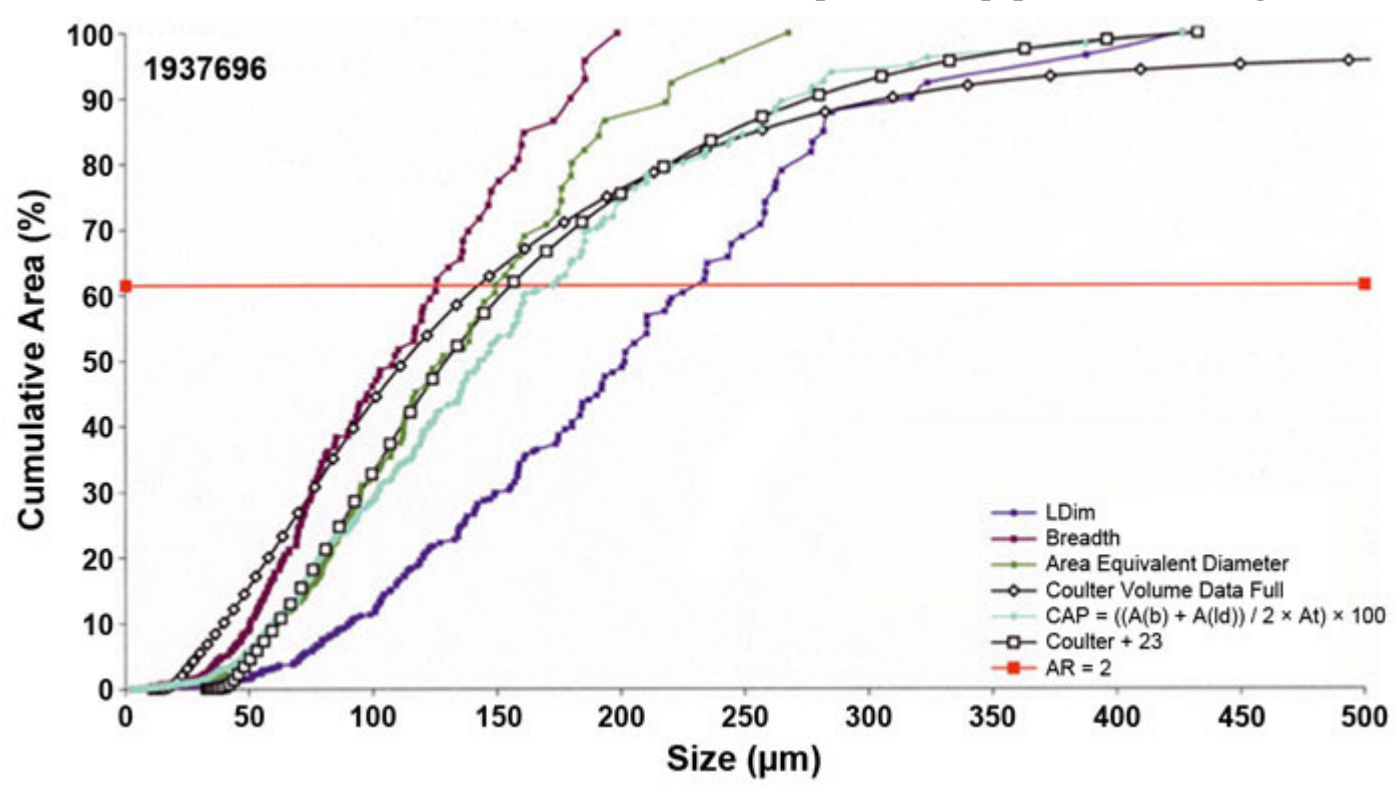

Figure 9. Example 1 of correction applied to \%V and IA plot: sample $1937696 \mathrm{a}$.

\section{AR Less Than 2: Nonrectangular Particles}

As the shape of particles becomes less rectangular, the LDIA relationship appears to become a function of a singledimensional descriptor (ie, Equation 1 does not apply) (see Figures 11 and 12). The Disk1 LD data corrected for agglomeration (upper integration limit set to $92 \%$ ) and bimodality (lower integration limit set to ca $10 \%$ ) is seen to be coincident with the IA "longest dimension" plot. The mica ECAD IA data corrected for bimodality (particles less than $20 \mu \mathrm{m}$ excluded from analysis) is seen to be coincident with the LD plot.

The LD-IA relationship's dependence upon a singledimensional descriptor for circular and nearly circular par- ticles is expected based upon the known assumptions within LD data analysis algorithms. That the single-dimensional descriptor should be "longest dimension" rather than ECAD is not necessarily expected from these assumptions, but it is acceptable. As discussed below, IA dependence upon particle orientation may be a factor in this result.

The LD-IA relationship's dependence upon a singledimensional descriptor for mica can be rationalized as the result of the absence of a contiguous longest dimensional diffracting boundary. The longest dimensional end of the average mica particle is triangular (see Figure 1). The diffraction from the longest dimensional chord(s) of the average mica particle therefore corresponds to diffraction from

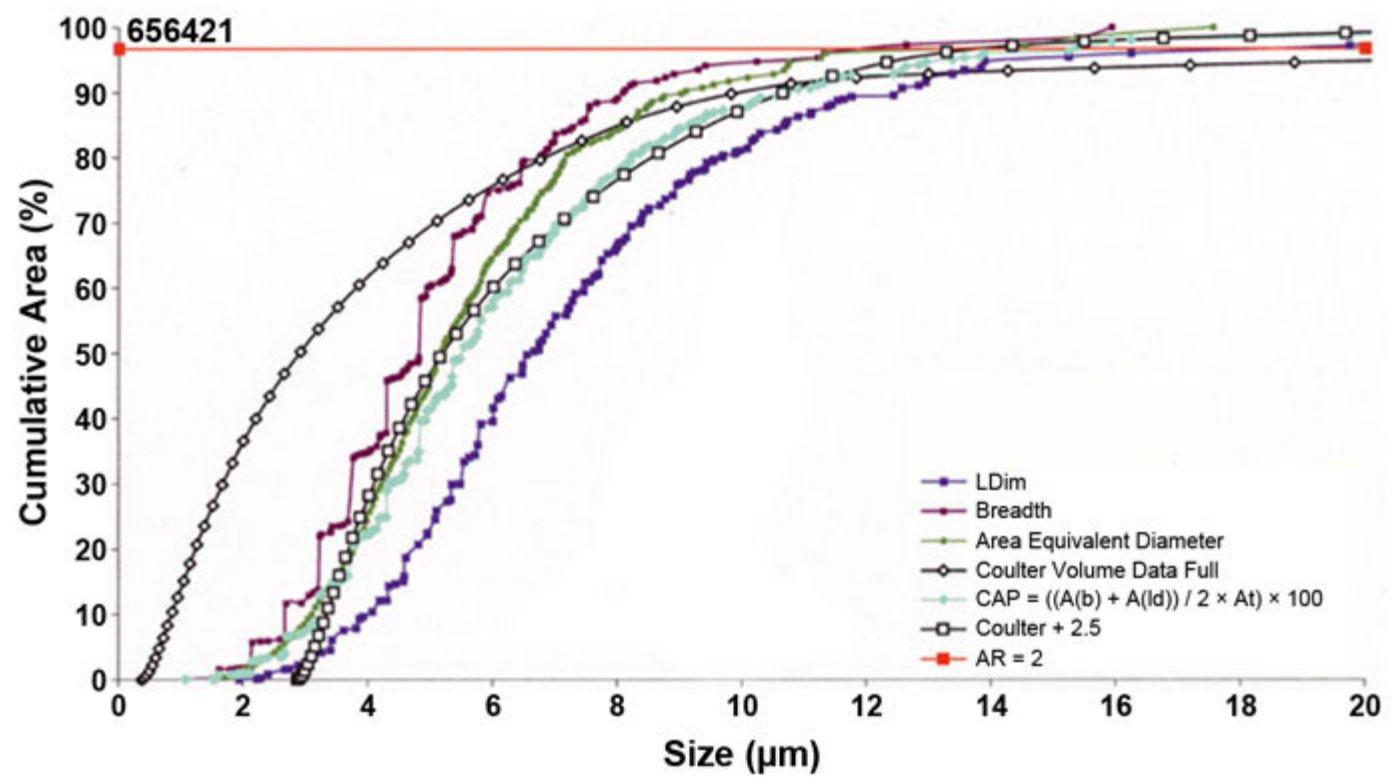

Figure 10. Example 2 of correction applied to \%V and IA plot: sample 656421. 
AAPS PharmSciTech 2006; 7 (3) Article 69 (http://www.aapspharmscitech.org).

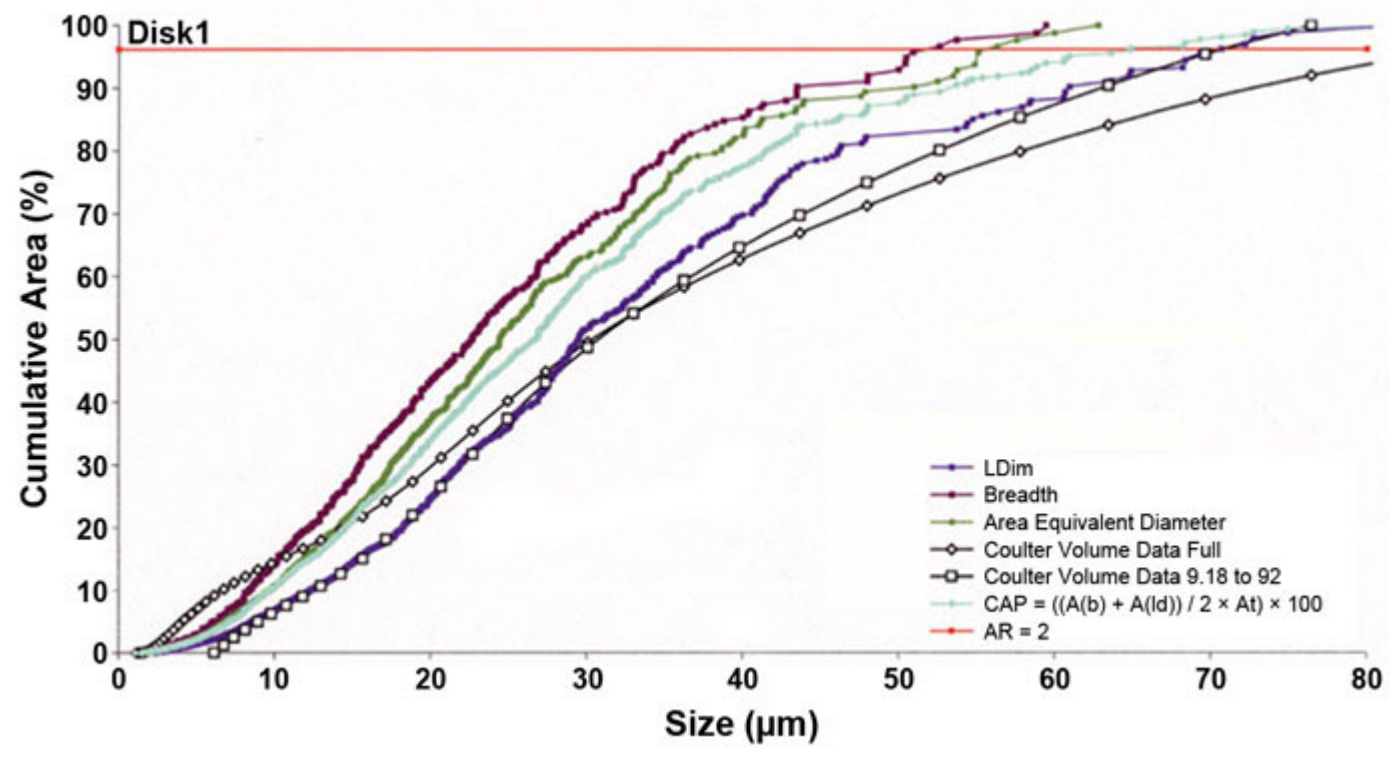

Figure 11. Example of LD \%V and IA plot for a bimodal, polydisperse, low-AR $(<1.5)$ elliptical sample (Disk1).

a point or a set of points that is small in number compared with that corresponding to diffraction from the breadth of the particle. This line of reasoning appears to be supported by the observation that the IA breadth and ECAD corrected data plots are both superimposable with the LD plot (see Figure 12).

\section{IA Particle Orientation Dependence}

IA dimensional measurements are by definition ${ }^{8,25}$ particleorientation dependent. In the course of microscope slide preparation of a sample for IA within the investigator's laboratory, additional sample dispersion typically is attempted by sliding the full-length microscope coverslip several times from front to back along the length of the slide.
This practice tends to orient nonspherical particles so that their longest dimension is parallel with the long axis of the slide.

As shown in Figure 13, preferential orientation often manifests itself in the IA/LD plots by

1. coincidence of the "maximum horizontal chord" and the "breadth" plots,

2. coincidence of either $X$ or $Y$ Feret $(A R>2)$ plot with the IA "Longest Dimension" plot, and

3. significant separation between the $\mathrm{X}$ and $\mathrm{Y}$ Feret plots.

Preferential orientation will influence the IA results of all nonspherical particles regardless of the sample's PSD width and average AR. This complicates the assignment of IA

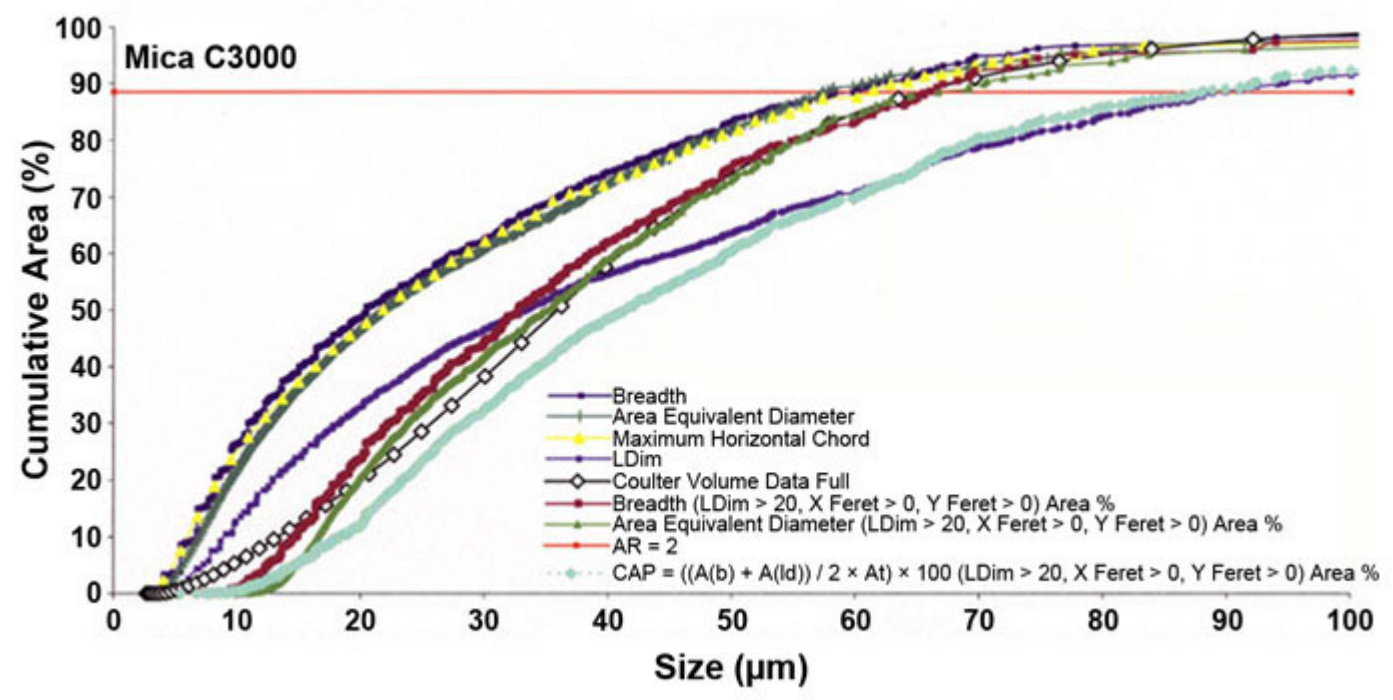

Figure 12. Example 2 of LD \%V and IA plot for a bimodal polydisperse low-AR $(<1.5)$ irregularly shaped sample (mica C3000). 
AAPS PharmSciTech 2006; 7 (3) Article 69 (http://www.aapspharmscitech.org).

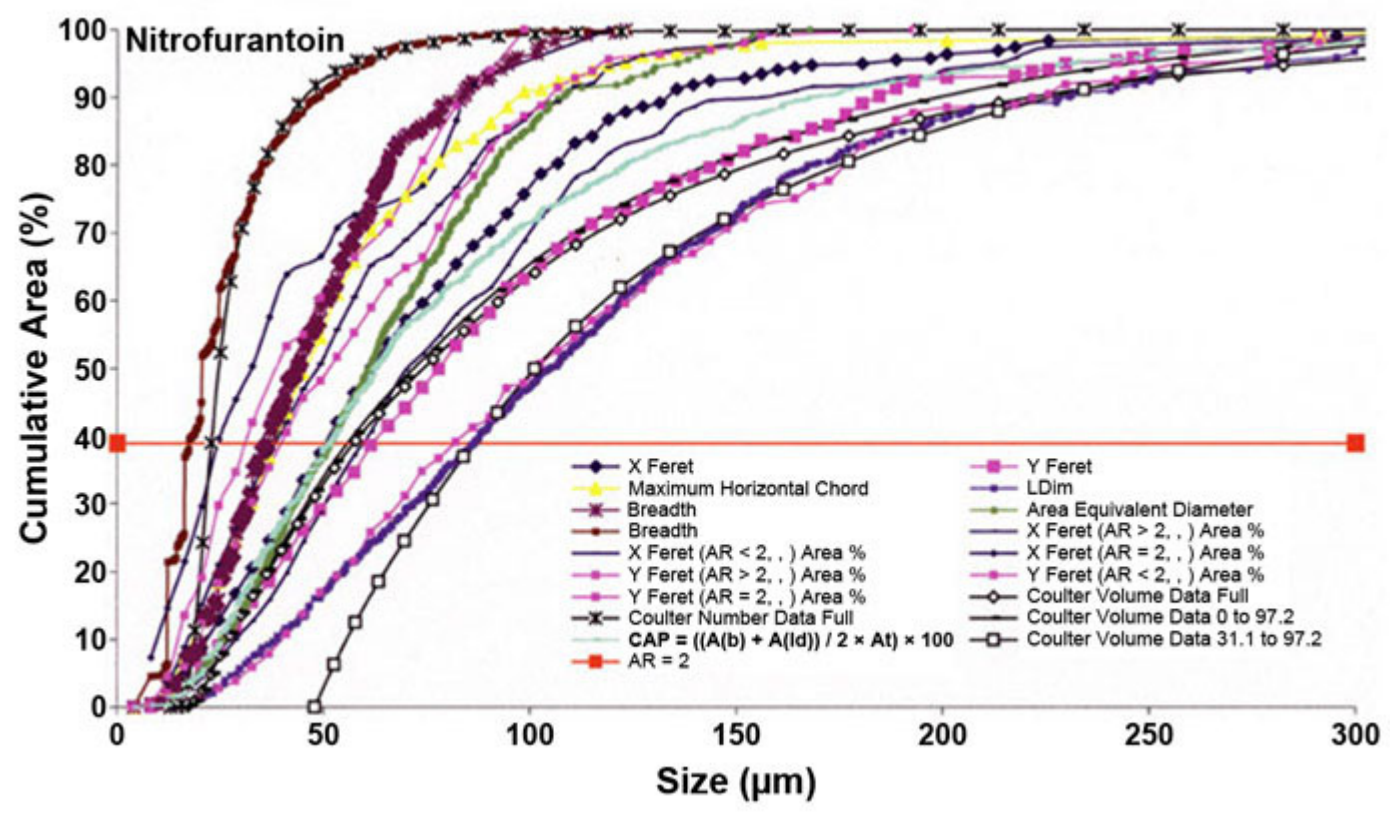

Figure 13. LD \%V and IA plot for nitrofurantoin showing possible influence of IA result orientation dependence.

dimensional descriptors to actual physical dimensions and is believed to be a partial factor influencing the extent to which the LD data are described by Equation 1.

\section{Effect of Multidimensional Character on LD Volume Statistics}

As seen from the above data, in the case of approximately rectangular particles the breadth contribution to the $\mathrm{LD} \% \mathrm{~V}$ psa reduces the values of the ESVD anticipated if only the longest dimensions of the particles are measured. Also, as the average AR increases, the breadth contribution leads to decreasing sensitivity of LD measurements to PSD broad- ening (see Table 2). This behavior explains results of many studies made over the past decade in which the LD \%V psa results from the measurement of high $\mathrm{AR}$ materials $(>2)$ were found to be smaller than the corresponding data from other psa techniques as, for example, in the work of Brewer and Ramsland for the needles described earlier. This finding is also supported by data from Mang et $\mathrm{al}^{18}$ in which a comparison was made of IA and LD psa results using both the standard analysis model based on the assumption of spherical particle symmetry and using a custom model for rectangular particles. They showed that even for particles with ARs as low as 1.51 the sphere-based LD psa results gave an under-sizing of the $\mathrm{d}_{\mathrm{v}} 50$ value. Clearly in the measurement

Table 2. Image Analysis and Laser Diffraction Response as a Function of Increasing Aspect Ratio and Particle Size

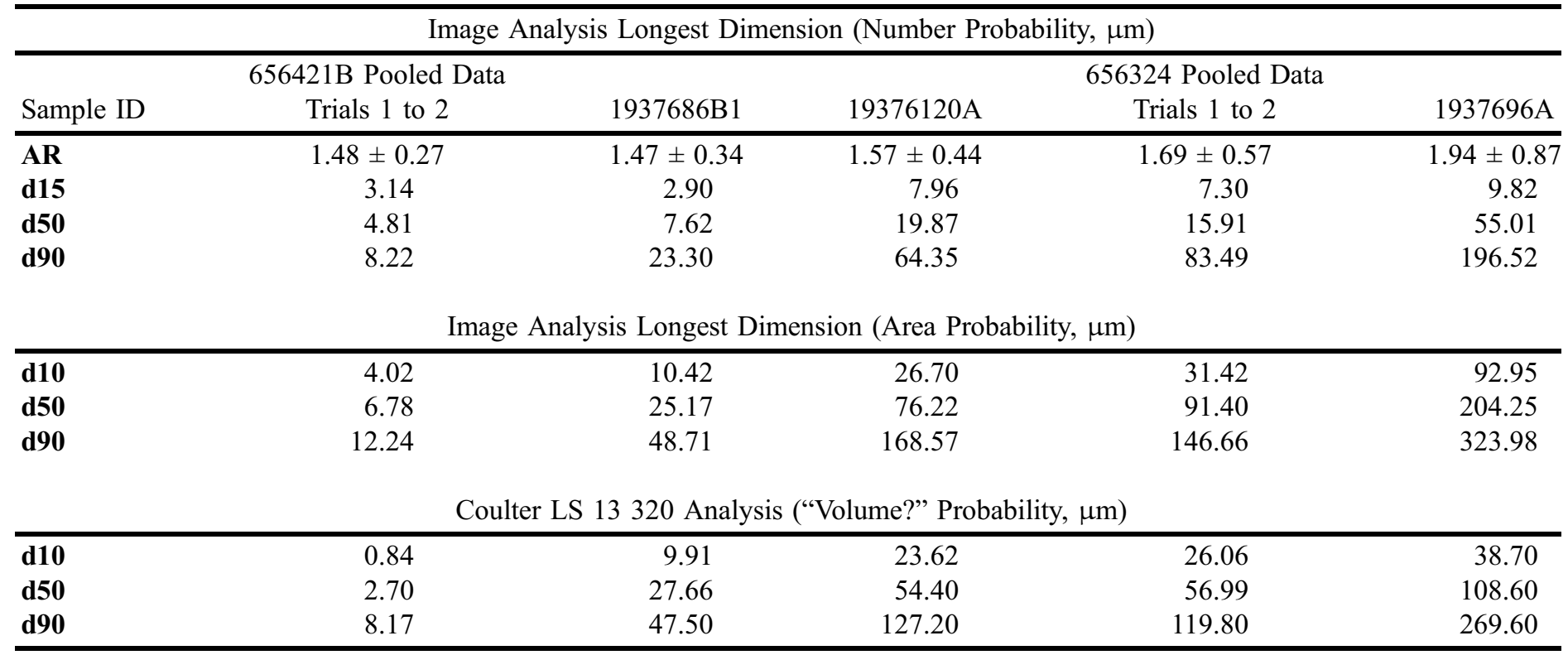




\section{AAPS PharmSciTech 2006; 7 (3) Article 69 (http://www.aapspharmscitech.org).}

of nonspherical particle systems, LD psa systems are not actually providing data in terms of an ESVD or ECAD but in terms of 2 or more dimensional descriptors as indicated by Equations 1 and 2.

\section{Image Analysis and Laser Diffraction Results Cross-validation}

The direct cross-validation of IA and LD psa results for nonspherical particles has been difficult over the past decade as a result of the absence of a means to quantitatively compare the data sets. The development of an Excel macro set fills that void to some degree as demonstrated in the above results. Using these direct graphical representations, it is easier to find correlation between LD and IA data representation, independent of the choice of a particular analytical representation of the nonspherical particle under investigation. This allows the development of a pragmatic choice of analysis parameters for both IA and LD, leading to a decision whether or not LD can be used for quality control (QC) and under what analysis conditions. The above examples demonstrate that Excel macro-assisted evaluation of the relationship between IA and LD psa data leads to an enhanced understanding of both IA psa and LD psa data.

\section{CONCLUSIONS}

The Excel macro-assisted graphical evaluation results demonstrate that IA and LD psa data obtained from the measurements of nonspherical particle systems can be successfully correlated when allowance is made for the limitations of the applicability of the ESVD and random particle orientation assumptions. Correlation has been shown to typically require more than a single IA size descriptor for particle systems characterized by ARs greater than ca 1.5. In the case of approximately rectangular polydisperse particle systems, it has been demonstrated that LD data can be approximately expressed in terms of IA size descriptors according to a simple variation of the law of mixtures in terms of the area-based breadth and longest dimensional fractional contributions to total measured particle system diffraction.

\section{ACKNOWLEDGMENTS}

The authors wish to gratefully acknowledge the support of JJPRD management, especially Daniel Korey, and to extend our thanks to Ogelbay Norton Specialty Minerals, Kings Mountain, NC, and the Engelhard Corporation, Iselin, NJ, for the donation of the mica samples used in this research effort.

\section{REFERENCES}

1. Kelly RN, Lerke SA. Particle size measurement technique selection within method development in the pharmaceutical industry. Am Pharm Rev. 2005;8:72-81.
2. Khalili MA, Roricht WL, Luke Lee SY. An investigation to determine the precision for measuring particle size distribution by laser diffraction. World Congress on Particle Technology 4; July 21-25, 2002; Sydney, Australia. Paper no 111.

3. International Organization for Standardization. (ISO) 13320-1; Particle Size Analysis - LD Methods, Part 1: General Principles. Geneva, Switzerland: ISO Standards Authority; 1999.

4. United States Pharmacopoeial Convention. $<429>$ Light diffraction measurement of particle size. In: Supplement to USP 27. Vol. 29, No. 4. In-Process Revision. Rockville, MD: United States Pharmacopeial Convention; 2003.

5. Bell R, Dennis A, Hendriksen B, North N, Sherwood J. Position paper on particle sizing: sample preparation: method validation and data presentation. Pharm Technol Eur. 1999;11:1-3.

6. Kelly RN, Kazanjian J. Commercial reference shape standards use in the study of particle shape effect on laser diffraction particle size analysis. AAPS PharmSciTech. 2006;7:E49.

7. Gabas N, Hiquily L, Laguuerie C. Response of laser diffraction particle sizer to anisometric particles. Part Part Syst Charact. 1994; 11:121-126.

8. Brewer E, Ramsland A. Particle size determination by automated microscopical imaging analysis with comparison to laser diffraction. J Pharm Sci. 1995;84:499-501.

9. Bowen P, Humphry-Baker R, Herard C. Particle size distribution measurement of regular anisotropic particles: cylinders and platelets. Proceedings of World Congress on Particle Technology 3; July 6, 1998; Brighton, UK.

10. Bowen P, Sheng J, Jongen N. Particle size distribution measurement of regular anisotropic particles: cylinders and platelets-practical examples. In: Recents Progres en Genie des Procedes. 2001: 251-256.

11. Bowen P. Particle size distribution measurement from millimeters to nanometers and from rods to platelets. J Dispersion Sci Technol. 2002;23:631-662.

12. Kaye BH, Alliet D, Switzer L, Turbitt-Daoust C. The effect of shape on intermethod correlation of techniques for characterizing the size distribution of a powder. I. correlating the size distribution measured by sieving, IA, and diffractometer methods. Part Part Syst Charact. 1997;14:219-255.

13. Kaye BH, Alliet D, Switzer L, Turbitt-Daoust C. The effect of shape on intermethod correlation of techniques for characterizing the size distribution of a powder. II. correlating the size distribution as measured by diffractometer methods, TSI-Amherst aerosol spectrometer, and Coulter counter. Part Part Syst Charact. 1999; 16:266-273.

14. Matsuyama T, Yamamoto H, Scarlett B. Theoretical prediction of effect of orientation on diffraction pattern transformation of diffraction pattern due to ellipsoids into equivalent diameter distribution for spheres. Part Part Syst Charact. 2000;17:41-46.

15. Brown DJ. Comments on "extending the use of particle sizing instrumentation to calculate particle shape factors. Powder Technol. 1986;49:71

16. Umhauer H, Bottlinger M. Effect of particle shape and structure on the results of single particle light-scattering size analysis. Appl Opt. 1991;30:4980-4986.

17. Endoh S, Kuga Y, Ohya H, Ikeda C, Iwata H. Shape estimation of anisometric particles using size measurement techniques. Part Part Syst Charact. 1998;15:145-149.

18. Mang JT, Skidmore CB, Kramer JF, Phillips DS. Quantitative 


\section{AAPS PharmSciTech 2006; 7 (3) Article 69 (http://www.aapspharmscitech.org).}

morphological characterization of high explosive crystal grains by light diffraction and microscopy. Paper presented at: Fraunhofer-Institut fur Chemische Technologie 31st International Conference; June 27-30, 2000; Karlsruhe, Germany.

19. Berthold C, Klein R, Luhmann J, Nickel K. Characterization of fibres and fibre collectives with common laser diffractometers. Part Part Syst Charact. 2000;17:113-116.

20. Zitoun KB, Sastry SK. Orientation distribution of solids in continuous solid-liquid flow in a vertical tube. Chem Eng Sci. 2004; 59:2767-2775.

21. Jianzhong L, Weifeng Z, Zhaosheng Y. Numerical research on the orientation distribution of fibers immersed in laminar and turbulent pipe flows. J Aerosol Sci. 2004;35:63-82.

22. Rawle A. The importance of particle sizing to the coating industry. I. Particle size measurement. Adv Colour Sci Technol. 2002;5:1-12.

23. Kippax P. Issues in the appraisal of LD particle sizing techniques. Pharmaceutical Technology Europe Web site. 2005; Available at: http:// www.ptemag.com/pharmtecheurope/data/articlestandard/pharmtecheurope/ 082005/147556/article.pdf. Accessed: February 11, 2005.

24. Prasanna HR, Jefferson EH, Taylor JS, Hussain AS, Karuhn RF, Lyon RC. Comparative analysis of common particle sizing techniques for pharmaceutical powders. FDA Science Poster 2001. Available at: http://www.particlesize.com/Bibliography/PS\%20FDA\%20SF01\%20Poster. pdf. Accessed: June 27, 2005.

25. Russ JC, ed. Image measurements. In: Computer-Assisted Microscopy: The Measurement and Analysis of Images. New York, NY: Plenum Press; 1990:175-218. 